\title{
Concepts of Trapping Topologically by Shell Molecules
}

\author{
MIECZYSLAW MACIEI EWSKI \\ Institute of Organic Chemistry and Technology \\ Warsaw Technical University \\ Warsaw, Poland
}

\section{A BSTRACT}

Concepts of the synthesis of shell topological compounds, which consist of a guest molecule (or molecules) trapped by a host molecule with a spacial, egg shell-like structure are discussed. Generally, both constructing the shell molecule in the presence of a guest molecule and constructing the guest molecule in the presence of the shell (host) are ways to "shell" topological ccinpounds. The preparation of shell molecules may consist of the completion of "preshell" molecules or of obtaining cascade branched oligomers and polymers. Cyclodextrins and substances like triquinance are considered to play a role in preshell molecules. Shell molecules may also be obtained by polyreaction of a monomer of the XRY ${ }_{n}$ type, which results in a cascade branched molecule of shell structure (spherical form). When the polyreaction is continued, the cascade branched mole cule becomes a "cast" one. It is theoretically possible to enclose a guest molecule inside the shell during the cascade branching process if there is a good solvent (of high expansion coefficient value) in respect to the growing branches. A spacially developed molecule of both "empty" and "cast" structure may be obtained also by the known "step by step" cascade branching process which Involves, for instance, a repeated cyanoethylation-reduction reaction. 
Some kinds of compounds with topological bonds [ e.g., Refs. 1-7] are known, and methods for their synthesis are available. However, up to now three types of the compounds, (catenanes, rotaxanes, and knots) have only been examined as their polymer derivatives. These have been based on macrocyclic molecules.

In the present paper new kinds of topological compounds and concepts of the synthesis of them are discussed. The topological compounds just mentioned consist of a guest molecule (or molecules) trapped by a host molecule, which is a spacially developed structure of completely closed form, like an egg shell. The term "shell topological compounds" adequately reflects such a type of structure. The molecular geometry of any solid figure, which is empty inside, can play the role of the host molecule in shell compounds. In the simplest case it would be a molecule of spherical structure.

One can draw at least two pathways which should lead to the synthesis of shell topological compounds. One of them includes the preparation of shell molecules in the presence of guest ones. It can be assumed that some guest molecules will be trapped inside. On the other hand, the preparation of guest molecules in the presence of an available shell is another pathway to the synthesis of shell topological compounds. In this procedure, the shell molecules used must exhibit selective permeability. They should be impermeable in respect to guest molecules and penetrable for substrate molecules suitable for synthesis. Such a situation allows the substrate molecules to penetrate inside of the shell and react there in special conditions to result in guest molecules. The latter are combined with the shell topologically. Both statistical and directed methods seem to be available for the synthesis of shell topological compounds. However, the preparation of shell molecules alone remains the most important problem in this synthesis. Two concepts of the synthesis of shell molecules will be discussed. The first one consists of the completion of the molecular geometrical form which can be considered as an initial part of the shell. These can be called preshell molecules. The second uses oligomer or polymer compounds of spacially developed structures. Low molecular preshell molecules will be useful in the synthesis of rather simple shell topological compounds (Fig. 1a, 1b, 1c), and the polymers will result in products of higher complexity (Fig. 1d, 1e).

Sokolov [8] has perceived the possibility of the synthesis of shell topological compounds by dimerization of triquinacene $[9]$ as a conplex with metals. Sokolov believes that this reaction would directly give the topological compounds of pentagonal dodecahedrane (up to now it has not been obtained) with metal atoms remaining in the free internal space of this spherical molecule. Besides that, it seems there is no information in the literature in which the synthesis of shell topological compounds is described [8]. It is very probable that the synthesis of pentagonal dodecahedrane will be accomplished shortly as a result of intensive investigations made by Paquette et al. $\mid 10-13]$ on derivatives of this compound which, however, do not have a complete 

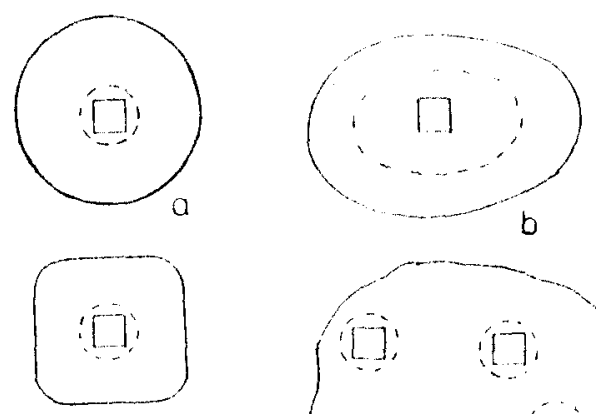

C
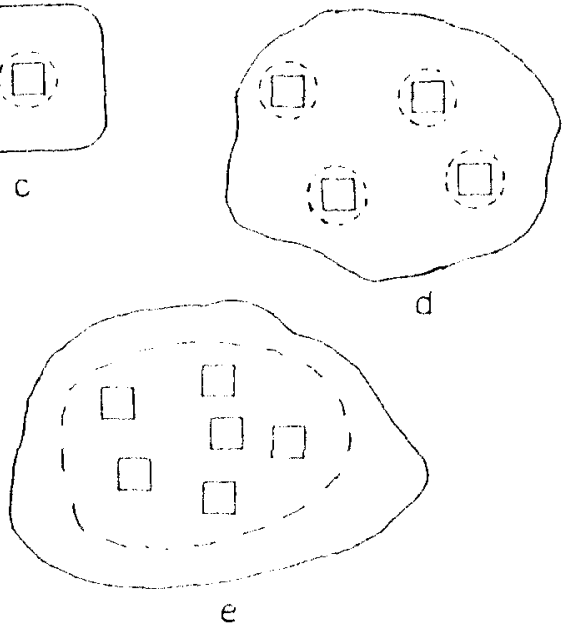

FIG. 1. Schemes of shell topological compounds: (--) external contours of shell molecules, (-) internal contours of shell molecules, (u) guest molecules.

spherical structure. This would give the possibility of the synthesis of topological compounds based on dodecahedrane. We can hope that other polyledranes will be available for topological synthesis in the future.

Cyclodextrins molecules are another type of preshell compound. They can place in their free spaces nut only atoms but also molecules of many compounds, especially organic ones. Inclusion componds of cyclodextrin are very well known. Cylindrical cyclodextrin molecules have two crowns of hydroxyl groups on their ends, and intramolecular cross-linking of each crown separately ("darning" of holes) can mean the completion of the shell. If guest molecules are put inside cyclodextrin ones, molecules of shell topological componud will be obtained as in Fig. 2. The small number of compounds available for the initial shell structure (practically, only the houndin:s of cyclodextrins, alpha, beta, and gamma, are now available) sibuificantly limits the pussibility of the eventual development of low molecular shell topological compounds. It seems that there are possibilities of 

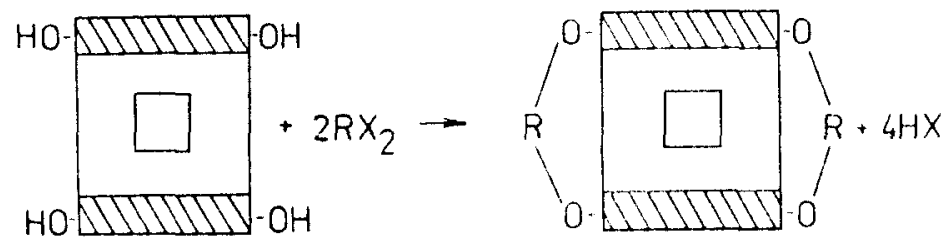

FIG. 2. Intramolecular cross-linking ("darning" of holes) of cyclodextrin molecule. Only 4 OH groups are shown instead of 18 , 21 , or 24 (for alpha-, beta-, or gamma-c yclodextrin, respectively).

the synthesis of more complex shell compounds in the field of polymers. This problem will be discussed.

The approach to the synthesis of macromolecular shell topological compounds does not include cross-linked systems. Topological compounds, which exist in the form of separate molecules, seem to he most interesting and will be discussed below. The main problem here is the synthesis of a shell molecule alone.

The protein cyst coating a virus nucleic acid is a very well known naturally occurring polymer shell "molecule" of almost full shell structure. This protective coat is this nucleic acid. It seems unlikely that we will soon be able to synthesize a polymer shell molecule with such a thin-wall structure. A special molecular matrix is surely needed for this purpose.

As will be shown below, a way to synthesize a macromolecular shell molecule or a macromolecular shell topological compound may consist of the synthesis of a macromolecule having a geometry of a cast space figure, e.g., ball, cylinder, cone.

The only way to synthesize cast macromolecules is by a polyreaction which is accompanied by an intensive chain branching process unless the reaction product is cross-linked. The well-known star or comb polymers are not adequate; it is necessary to synthesize cascade branched polymers.

There is a kind of polyfunctional monomer (more than two functional groups) which does not result in cross-linked polymers even at a high degree of polyreaction. These monomers have the general formula $\mathrm{XRY}_{\mathrm{n}}$ in which the polyreaction can be carried out only between different functional groups. " $n$ " is a whole number greater than 1. In such monomers $X$ can be, for instance, either a hydroxyl or carboxyl group, and $Y$ can be either a corresponding carboxyl or hydroxyl gronp. The greater " $n$," the greater the degree of branching in the polymer chain.

Flory $[14,15]$ was first to pay attention to such monomer types, and he derived some statistical relations in polyreactions of them. 
He also indicated several examples of cascade branched polymers in the literature. Cascade branched structures, described mainly nuthematically (theory of branching processes, graph theory), have served for many years as models (tree-like) to explain network formation and properties of cross-linking systems [e.g., Refs. 15-24].

From monomers of simplest type XRY $\mathrm{X}_{2}$, two kinds of polymer structure can be obtained in extreme cases.

a. Linear Structure<smiles>[Y][Y]([Y])[R]([Y])[Z]([Y])[R]([Y])[Z]([Y])[Y]</smiles>

where $\mathrm{Z}$ is a new group resulting from the reaction between $\mathrm{X}$ and Y.

b. Cascade branched structure shown below is the initial polyreaction product

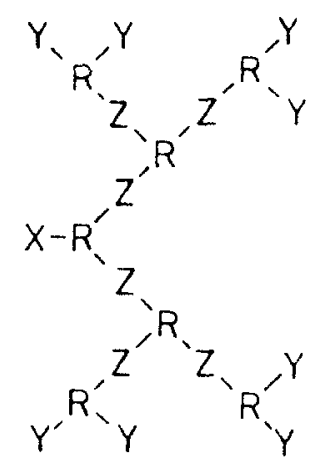

The creation of a linear structure will be preferable in this case when the $\mathrm{Y}$ groups in the monomer molecule have different reactivities. The equivalence of all $Y$ groups will give rise to the predoninance of the branched structure. A perfect cascade branched structure would have the polymer molecule growing in all directions at the same rate. Deviation from a perfect cascade branched structure will be of statistical nature as well as coming from the steric isolation of the reacting groups. In many cases it seems possible to determine the cascade structure content in the reaction product by chemical analysis and spectroscopy. The cascade structure content can be express by the rolation of the amount of $-R=\frac{Y}{Y}$ groups (when $n=2$ ) to the amount of $-R=\frac{Z}{Y}$ 
groups. The first formula represents a cascade branched structure and the second represents a linear one. In many cases both kinds of groups should be distinguishable chemically and spectroscopically. A perfect cascade branched structure will be represented only by $-\mathrm{R}=\mathrm{Y}$ groups distant from the initial mer of the polymer clain by the same amount of chemical bonds. Analogously, one can consider the situation for monomers of $n$ more than 2 .

The probability of creating of cast molecules in cascade branching polyreaction comes directly from so-called "Malthusian packing paradox" $[21,22,24]$. It tells us that the space available for a cascade branched growing molecule (tree-like structure) cannot accommodate all structural units when all functional groups react. The Malthusian packing paradox, which is related to the gel of crosslinking systems, is reflected by the situation where the number of structural units at the $r$ bond distance from a given unit in the gel structure is proportional to $\gamma_{v}{ }^{r}$, while the space available for these units is proportional to $\mathbf{r}^{2}[21] . \gamma$ denotes the relative conversion which, exceeds 1, after the gel point then is $\gamma^{r} / r^{2}-\infty$. If shell topological compounds are involved, it is useful to note how the relation of the volume needed for accommodating the structural units to the volume available for them changes depending on the degree of polymerization of a growing molecule. This relation will express the density of packing of a growing molecule in the space available for it.

Let a simple monomer $\mathrm{X}-\mathrm{CH} \mathrm{Y}_{\mathrm{Y}}^{\mathrm{Y}}$ condense with a molecule $\mathrm{X}-\mathrm{Y}$ as a by-product of the reaction. A perfect cascade branched polymer is assumed to be created:

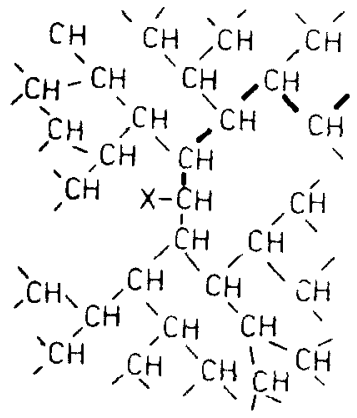

This structure, shown schematically on the plane, is embedded in space, of course. A cast molecule will be produced on condition that the structural units of the polymer $(\mathrm{CH})$ fill a ball volume determined 
by a radius, which equals the length of a branch, being extended to a maximum. This volume is the largest that can be available for that molecule (the lengths of each branch are equal in a perfect cascade branched molecule obtained by polyreaction of XRY ${ }_{n}$; one of them is indicated by boldaced type in the formula.

Structural units of the polymer molecule must be considered as physical bodies having real dimensions. The CH group takes a volume of $10.9 \AA^{3}$ in the hydrocarbon molecule $\{25\}$. In Table 1 are results of calculations describing the perfect cascade branched polymer constructed from $C I I$ units, depending on the polymerization degree $m$ of a branch of the molecule. The $m$ value corresponds to the amount of $\mathrm{C}-\mathrm{C}$ bonds in a branch ( $\mathrm{m}$ also corresponds to the conventional numbering of generations in the theory of branching processes). $z$ (Column 2) denotes the amount of $\mathrm{CH}$ units in a whole polymer molecule. For a monomer $\mathrm{XCHY}_{2}, z$ increases in a geometrical progression during the polyreaction. For $m$ equal to $1,2,3,4$, etc., $z$ equals $(1+2),+(2 \times 2),+(2 \times 2 \times 2),+(2 \times 2 \times 2 \times 2)$, etc, respertively. The sum of such a progression is expressed by

$$
z=1+a, \frac{1-q^{m}}{1-q}
$$

where

$$
a_{1}=4=2
$$

The equation is common for monomers of the XRY ${ }_{n}$ type and then $a_{1}:=q=n$. In Column 3 there are length values $\ell$ of a branch in its most extended position. I' denotes the distance between the center of a carbon atom in an initial mer and the center of a bond following the CII group considered as the last one in the branch. For simplicily the end groups ( $\mathrm{X}$ and $\mathrm{Y}$ ) have been neglected and the molecule considered is issumed to be a ball section of radius $l$ in respect to some greater one, both being in concentric positions. This allows all bonds to be equivalent. On the base of $m, 2$, and $\&$ values, the volume $V_{k}$ (Column 4 ) of a ball determined by radius $\ell$ and the volume $V_{p}$, which is the sum of the volumes of all $\mathrm{CH}$ units within a ball section, can be calculated. In the perfect cascade branched molecule, no structure unit can appear outside that ball section and then $V_{p}$ will simply be equat to $\mathrm{zV}_{\mathrm{CH}}$. The relation $\mathrm{V}_{\mathrm{p}} / \mathrm{V}_{\mathrm{Q}}$ expresses the density of packing $\mathrm{k}$ in the molecule. In other words $\mathrm{k}$ indicates tie extent to which structural units fill the volume availatsle for a polymer molecule.

It must be emphasized that there is a specific packing density dependence on the degree of polymerization. With the excrption of 


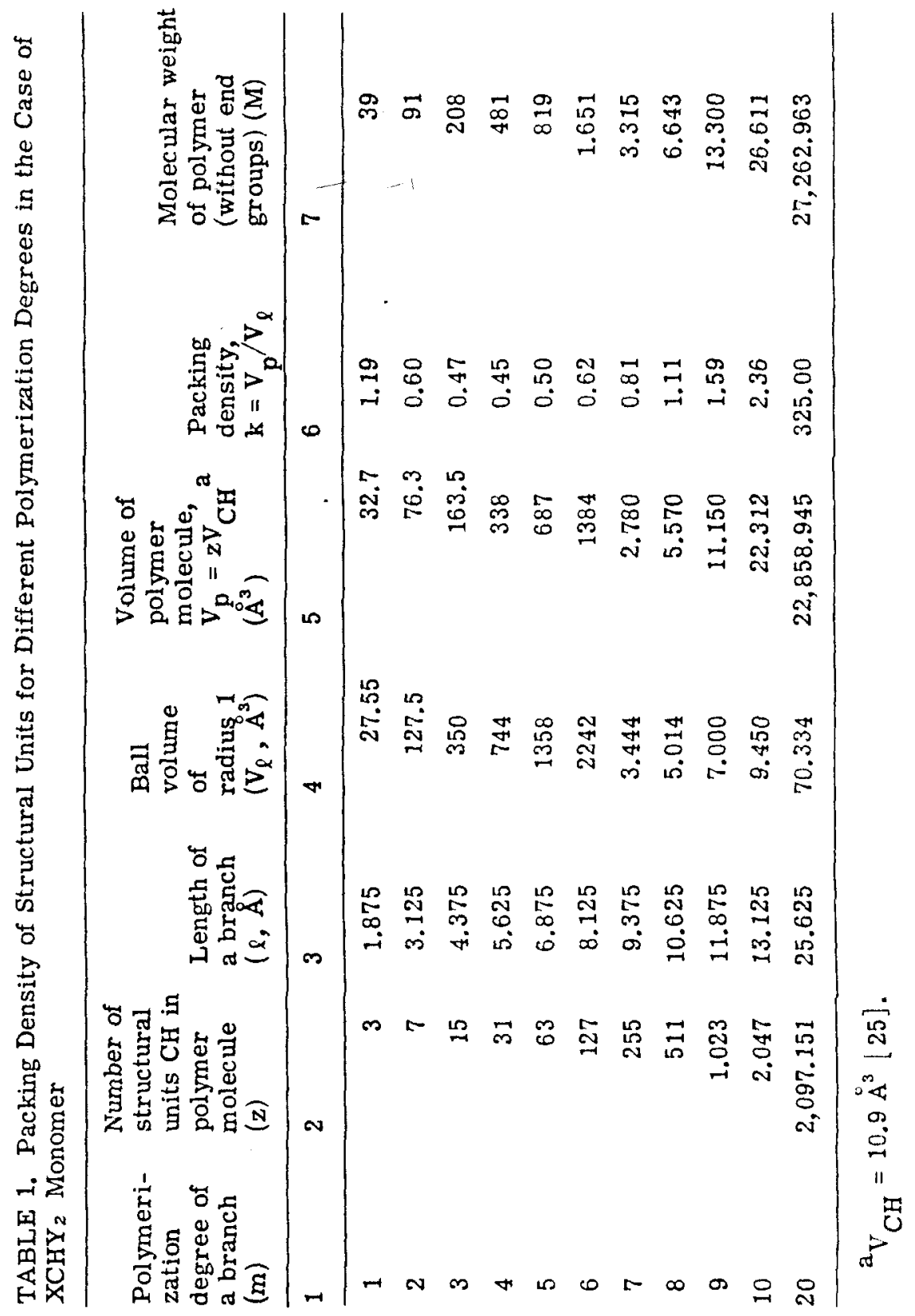


TABLE 2. Packing Density of Structural Units for Different Polymerization Degrees in the Case of $\mathrm{XCY}_{3}$ Monomer ${ }^{\mathrm{a}}$

\begin{tabular}{rrrcccr}
$\mathrm{m}$ & $\mathrm{z}$ & $\mathrm{l}$ & $\mathrm{V}_{1}$ & $\mathrm{~V}_{\mathrm{p}}=\mathrm{zV}_{\mathrm{c}}^{\mathrm{b}} \mathrm{k}=\mathrm{V}_{\mathrm{p}} / \mathrm{V}_{1}$ & \multicolumn{1}{c}{$\mathrm{M}$} \\
\hline 1 & 4 & 1.875 & 27.55 & 22.12 & 0.80 & 48 \\
2 & 13 & 3.125 & 127.5 & 71.9 & 0.56 & 156 \\
3 & 40 & 3.375 & 350 & 221.2 & 0.63 & 480 \\
4 & 121 & 5.625 & 744 & 669 & 0.9 & 1,452 \\
5 & 364 & 6.875 & 1,358 & 193 & 1.4 & 4,368 \\
6 & 1,093 & 8.125 & 2,242 & 6,044 & 2.7 & 13,116 \\
\hline
\end{tabular}

\footnotetext{
a Meaning of symbols see Table 1. ${ }^{b} V_{c}=5.53 A^{3}[25]$.
}

the case in which the molecule consists of three structural units and probably its shape strongly differs from a ball, the packing density is small and $\mathrm{CH}$ groups fill only $50 \%$ of the available volume at low degree of polymerizations $(m=2 / 5)$. When $m$ increases, the packing density becomes larger and larger and reaches quickly a value which is larger than the theoretically possible one. At $m=8(k=1.11)$ the perfect cascade branched molecule is indeed a cast one. It is certain that the polymer molecule becomes a cast ball even earlier than in this case because substances never reach a packing density equal to 1 , even in the crystalline state. A packing density of 0.80 is the limit, and this is not attained by crystalline polymers $|26|$. It scems reasonable to accept this value (in the absence of another) as a limit which a cast molecule may approach. One can also calculate the molecular weight desired for the cast molecule to occur. In the above case the polymer has reached a cast structure by $m=7$ (molecular weight about 3300).

Table 2 contains data on the perfect cascade polyreaction of a monomer with a larger number of the functional groups ( $3 \mathrm{Y}$ groups). In this case the reaction product surpasses the limiting value of packing density by $m=4$ (molecular weight 1452) when $X-Y$ molecules evolve as by-products. By the way, the reaction product is a variety of "diamond" structure (if the endgroups are neglected). As in diamonds, only quaternary carbon atoms are present in the molecule (angles and distances are neglected). Undoubtedly it would be interesting to obtain and determine the properties of such a "diamond" poiymer.

In any case, the perfect cascade branching process is possible for monomers $\mathrm{XRY}_{\mathrm{n}}$ only up to real limiting value of packing densily. 

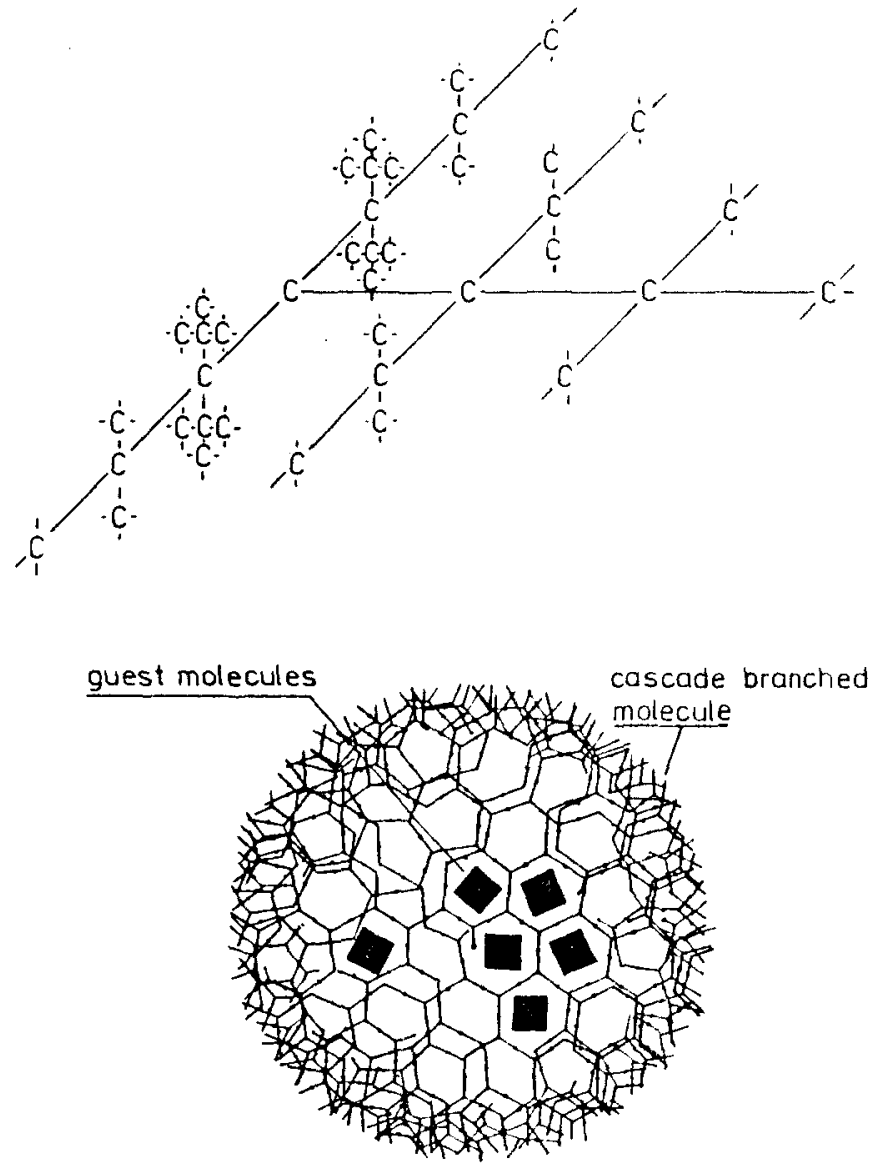

FIG. 3. Approximate distribution of packing density in a growing ball molecule.

The cascade process of some branches will be interrupted above this limit because of crowding. However, the molecule can continue to develop centrically in the form of a growing macroball while remaining a part of functional groups in its interior. One must remember that functional groups $\mathrm{Y}$ crowd mainly on the surface of the macroball in a growing molecule. This introduces a disturbance into the branching process. If the $\mathrm{Y}$ group volume is greater than that of the structural unit, the mathematical description of the branching process will be more complex.

Coming back to molecules with a low degree of polymerization (at low numbers of generations), it is easy to see that there is a situation 
at favorable conformations, when a partially unfilled ball molecule comes into being at the beginning of the reaction (see $k$ values at different values). This molecule becomes more and more filled wilh structural units (mainly in its yeriphery) in the next stops of the reacion, so that to be completely full at last. Before the molecule has become a cast one, there must be a moment at which the molecule has a cast structure only in its periphery, thus creating a shell which encloses a partially unfilled interior (Fig. 3). That is, a shell molecule has been oblained. Such an "empty" molecule can exist only hypothetically, of course, because the growing cascade branches will also fi]l the initial free space. It is, however, theoretically possible for space unfilled with struclural units to remain if this space is filled with guest molecules before the shell is completed. Then it is possible to obtain a shell topological compound directly. There will be especially favorable conditions if the cascade polyreaction proceeds in the presence of guest molecules which are a good solvent for the polymer. A good solvent means a solvent with a sulficiently great so-called average expansion coefficient. In such a condition the growing branches will take an expanded confornation and the solvent molecules attracted by the polyner segments will be closed by the external shell of the growing ball (Fig. 3). This seems to be a very real pathway to the synthesis of shell topological compounds of the spherical type.

The analysis of the possibility of the synthesis of spherical topological compounds in the polymer field has been carried out for the simplest types of monomers. However, in practice the avajable monomers are usually more complex, and the functional groups are distant from each other. Some monomers with a more real structure will now be discussed. Data in Table 3 shows that if the functional groups of a monomer are distant, the limiting value of packing density will be reached by a polymer with a higher degree of polymerization. With 3,5-Diaminobenzoic acid (or acid chloride) as a monomer ( $x_{\text {unit }}-6.04 X$ ), the limiting packing density will not be surpassed until $m=13$. On the other hand, the polymer can become a cast molecule at a lower $m$ if the volume of a monomer molecule increases although $x^{\prime}$ remains constant. Suilable substituents can be introduced for this purpose either into a monomer molecule or into a polymer molecule. 3,5-diaminotrimellitic acid will give a cast structure at $m=11$. Thus there are many possibilities for the synthesis of cast ball polymers with various chemical structures and molecular weights.

It is rather hard to predict theoretically what part of a "cast" macromolecule will constitute free space and be taken by gutsit molecules. In the shell topological compound, part of the packing density will he due to the guest molecules, and then limiting value of the density calculated as above will be attained at lower degree of polymerization ( $V$ will be decreased by the total volume of the guest
molecules). 
虫

ญ

$\stackrel{ \pm}{ \pm}$

5

d.

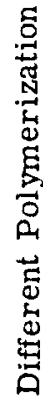

sั

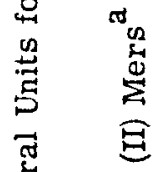

亭

总

㟧

总 $\frac{T}{2} \frac{T}{2}$

音

mं

a

要

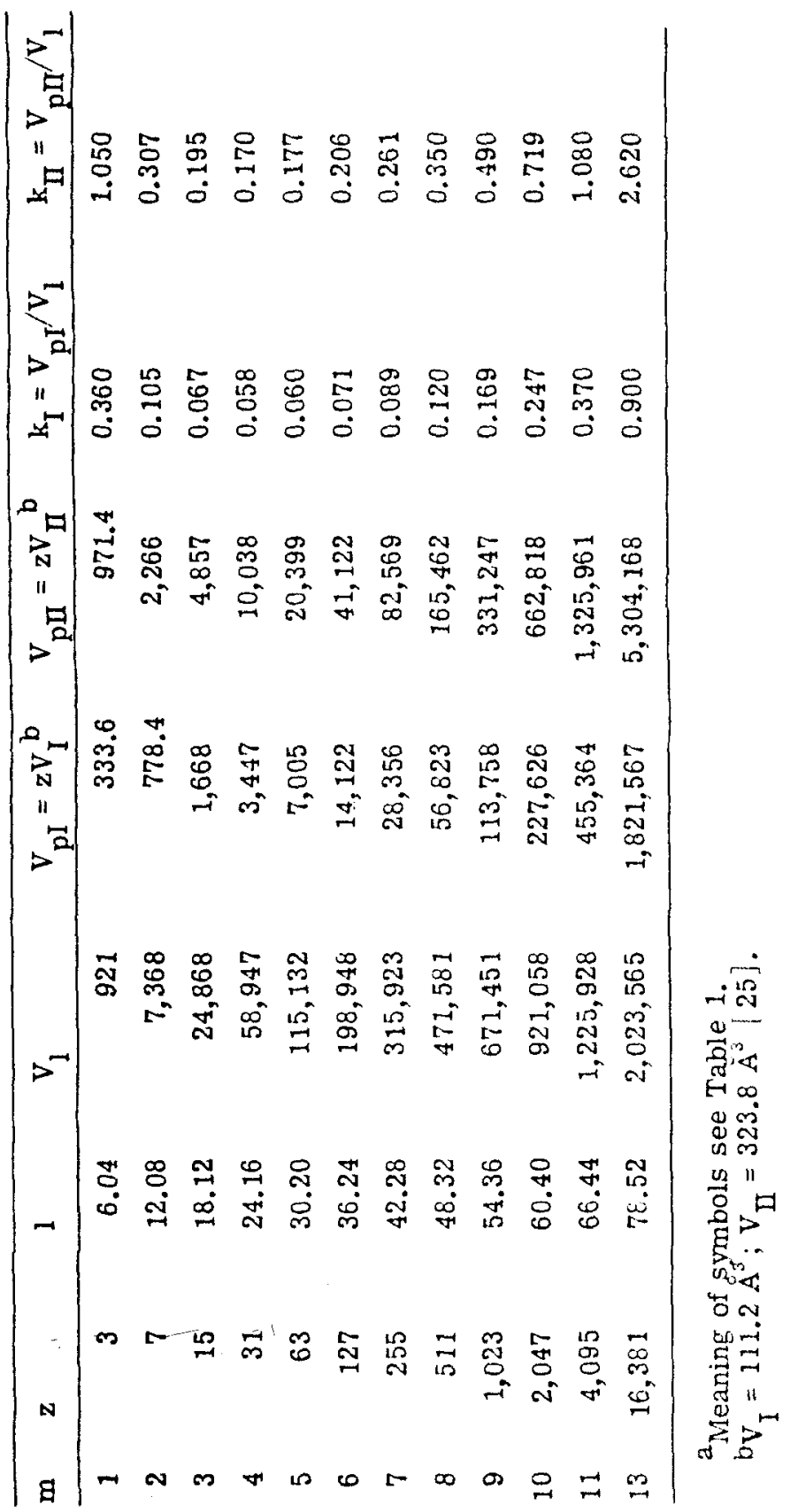


Cast macromolecules as well as shell topological compounds should exhibit some shape stability and undergo neither contraction nor expansion. For such molecules obtained in real conditions, the molecular weight distribution as well as the molecular shape distribution will be characteristic. Introduction of the term "average shape" for these molecules seems necessary. When the product of a perfect cascade polyreaction of $\mathrm{XRY}_{\mathrm{n}}$ is ball-shaped, that can be the average shape only in real conditions. The shape distribution of cascade branching molecules will be complex because of the possibility of the reaction of growing molecules with each other in addition to the reaction between growing molecules and the monomer.

However, there is the possibility of the synthesis of model spherical compounds of strictly ball shape by way of a "step by step" reaction. Low molecular weight cascade branched molecules, which have topologically shaped cavities or pseudo-cavities obtained in this way, have been described [27]. The authors guess they have properties of cryptands and are capable of binding ionic guests or molecules (as complex or inclusion compounds). According to Ruf. 27, cascade branched molecules have been obtained by several reputitions of two reactions: cyanoethylation of amino groups (each amino group can be substituted for by two cyanoethyl groups), and reduction of nitrile groups to amino functions. As a result of repeated reactions, polyaza molecules with "increasingly growing cavity size" (as the authors say) are obtained. What the authors really obtained under the conditions they used can be calculated. If even the largest polyaza molecules $\left(\mathrm{n}=2, \mathrm{~m}=3, \ell_{\text {unit }}=4.7, \mathrm{~V}_{\text {unit }}=49.71\right)$ are involved, their packing density $(0.063)$ is very far from that of cast structure and the molecules can be considered only as the octopus type [27]. However, the pathway indicated by the authors must lead to cast molecules at higher degrees of polymerization. It can be calculated for this system that the cast structure will be reached at $m=13(k=0.85)$. This means that the cyanoethylation reduction reactions would have to be repeated 13 times. This is a very laborious, time-consuning pathway to cast structures, but the only one if a perfect cascade branched structure is to be obtained. This is because no more than one monomer unit can be annexed on each branching chain at each step of the reaction. On the other hand, a very high yield reaction can be chosen which allows the units to be annexed by (practically) all the branching chains at each reaction step. Under these conditions the cast mole. cules of perfect balls form or the corresponding topological compound will be obtained after the real limiting density packing value is reached. The "step-by-step" concept of the synthesis of cast macromolecules is also useful in respect to the variety of geometrical forms of molecules created depending on the shape of the starting molecule. A last instance is a linear polymer with functional groups along the chain. If a polyvinylamine molecule undergoes repeated cyano. ethylation-reduction reactions, a cast molecule of cylinder form will 
be obtained. For such a molecule to be obtained it is enough for each mer of the polymer chain to be cascade branched to an extent which allows a unit cylinder (of a height equal to the mer length and radius equal to $\ell$ ) to be filled by the branches. If polyvinylamine is involved, simple calculations show that the packing density of the resulting polymer will pass the limiting value at $m=6$. The cast macromolecule created will have a cylinder form of about $50 \AA$ diameter. In this case there is a theoretical possibility of the synthesis of the topological compound which is represented by the polymer geometry of a pipe (as a shell molecule) filled with guest molecules by analogy to the compound geometry of the ball. The branching process must be carried out, of course, up to the moment when the ends of polyner pipe have been closed by the creation of the respective hemispheres. However, open pipe molecules would also be very interesting struclures from the inclusion chemistry point of view. Similar modification of polymers can also be carried out by polyreaction of monomer $\mathrm{XRY}_{\mathrm{n}}$ in the presence of a polymer which has $\mathrm{Y}$ functions of the same (or higher) chemical activity. However, as a result, a mixture of a modified starting polymer and spherical ones (created by the polyreaction of $X_{R Y}$ alone) will be most promising.

Following the procedure described above, one can synthesize many polymer structures based on copolymers as well as normally branched chains such as star and comb polymers. Many other low molecular weight and polymer structures are suitable as base substrates.

Experimental studies of these structures are underway.

\section{REFERENCES}

[1] E. Wasserman, J. Am. Chem. Soc., 82, 4433 (1960).

[2] G. Schill, Chem. Ber., 100, 2021 (1967); Habilitation Thesis, Universify of Freiburg, 1964.

[3] G. Schill, Catenanes, Rotaxanes, and Knots, Academic, New York, 1971 .

[4] B. Hudson and J. Vinograd, Nature (London), 216, 647 (1967).

5] G. Karagounis and J. Pandi-Agathokli, Prakt. Akad. Athenon, pp. $118-126$ (1970).

[6] M. Maciejewski and G. Smets, Paper Presented at 2nd Conference on Modification of Vinyl Polymers, Wroclaw, Poland, 1973; Sci. Papers Inst. Org. Technol. Plast., Wrocław Tech. Univ., 16, 57 (1974); Chem. Abstr., 83, 43826e (1975).

[7] M. Maciejewski, Habilitation Thesis, Warsaw Technical University, 1980.

[8] V.J. Sokolov, Vviedeniye v teoreticheskuyu stereokhimiyu, "Nauka," Moseow, 1979, p. 176. 
[9] R. B. Woodward, T. Fukunada, and R. C. Kelly, I. Am. Chem. Soc., 86, $3162(1964)$.

[10] L. A. Paquette, M. J. Wyvratt, O. Schallner, J. L. Muthard, W. J. Begley, R. M. Blankenschip, and D. Balogh, J. Org. Chem,s 44, 3616 (1979).

[11] L. A. Paquette, W. J. Beg]ey, D. Balogh, M. J. Wyvratl, and D. Bremner, Ibid., 44, 3630 (1979).

(12] R. L. Sobe zak and L. A. Paquette, Bid., 44, 4886 (1979).

13] L. A. Paquette, J. L. Muthard, and T. Cynkowski, J. Am. Chem. Soc., 101, 6991 (1979).

14] P. J. Flory, J. Am. Chem. Soc., 74, 2718 (1952).

15] P. J. Flory, Principles of Polymer Chemistry, Cornell University Press, Ithaca, New York, 1953.

16] W. H. Stockmayer, J. Chem. Phys., 11, 45 (1943).

17] I. J. Good, Proc. Cambridge Pli11os. Soc., 45, 360 (1943), through Ref. 19.

18] I. J. Good, Proc. R. Soc. A, 272, 54 (1963).

19 G. R. Dobson and M. Gordon, J. Chem. Phys., 41, 2389 (1964).

[20] M. Gordon and G. N. Malcolm, Proc. R. Soc., A, 295, 29 (1966).

[21] M. Gordon, T. C. Ward, and R. S. Withney, in Polymer Networks. Structure and Mechanical Properties (A. J. Chompir and S. Newman, eds.), Plenum, New York, 1971.

22] M. Gordon, Pure Appl. Chem., 43, 1 (1975), through Ref, 24.

23] M. Gordon and W. B. Temple, in Chemical Applications of Graph Theory (A. T. Balaban, ed.), Academic, London, 1976.

[24] K. Dusek, Makromol. Chem. Suppl., 2, 35 (1979).

[25] G. L. Slonimskii, A. A. Askadskii, and A. J. Kitaigordskii, Vysokomol. Soedin., A, 12, 494 (1970).

[26] B. Wunderlich, Fizika Makromolekul, Izd. Mir, Moscow, 1976.

27] E. Buhleier, W. Wehner, and F. Vögtle, Synthesis, p. 155 (1978).

Accepted by editor February 13, 1981

Received for publication March 6, 1981 